\title{
Deviatoric section shape functions for materials exhibiting dependence on shear-to-axial yield stress ratio
}

\author{
Aleksander Szwed ${ }^{1, *}$ \\ ${ }^{1}$ Warsaw University of Technology, FCE, ul. L. Kaczyńskiego 16, 00-637 Warszawa, Poland
}

\begin{abstract}
Proposal of a class of smooth functions describing shape of the deviatoric section of the failure surface of isotropic materials is given. Their convexity is investigated and the resultant restrictions to the free parameters are derived. Applications to pressure sensitive or insensitive materials as yield criteria are shown. Discussion of calibration of material parameters is carried out and dependence of the failure surface on the shear-to-axial yield stress ratio is demonstrated.
\end{abstract}

\section{Introduction}

Each phenomenon of yielding, damage or failure of materials, including cast iron, porous metals, metallic foams is quite complex and is the subject of an intense research. For example, behaviour of the mentioned materials depends on the first (pressure sensitivity) and third (Lode dependence) stress invariants. To formulate mathematical models for the aforementioned phenomena an approach based on the yield, damage or failure criteria is used, predominantly relying upon the experimental evidence of material behaviour.

In the present article proposal of new functions describing shape of the deviatoric section of the failure surface is given. Yield or failure criteria are tailored to interpolate typical experimental results for certain class of isotropic materials. The regarded shape functions used for formulation of the criteria preserve smoothness and convexity of the yield surface. Moreover, they can be included in the constitutive models of materials in which apexes or intersection corners exist, for example in case of the Drucker-Prager yield surface.

Excellent review of the shape functions for deviatoric section is given in [1]. Special case of functions analysed herein was introduced by Drucker [1, 2], but generalization was proposed in $[3,4]$. This paper reports further development of that type of shape functions.

\footnotetext{
* Corresponding author: a.szwed@il.pw.edu.pl
} 


\section{Shape functions of deviatoric section}

The analysis is restricted to isotropic materials, therefore the Haigh-Westergaard representation of the yield surface is used. The following class of isotropic scalar-valued functions is regarded:

$$
f(\xi, r, \Theta)=f\left(\xi, \frac{r}{g(\Theta)}\right)
$$

where $\xi, r$ and $\Theta$ are invariants of stress tensor $\boldsymbol{\sigma}$ defined as:

$$
\xi=\frac{1}{\sqrt{3}} \operatorname{tr} \boldsymbol{\sigma}, \quad r=\|\mathbf{s}\|=\sqrt{\operatorname{trs}^{2}}, \quad \Theta=\frac{1}{3} \arccos \left(\frac{\sqrt{6} \operatorname{trs}^{3}}{\sqrt{\operatorname{tr}^{3} \mathbf{s}^{2}}}\right),
$$

and $\mathbf{s}=\boldsymbol{\sigma}-\xi \mathbf{k}, \mathbf{k}=\mathbf{I} / \sqrt{3}$, in which $\mathbf{I}$ is the second order unit tensor. A shape function $g(\Theta)$ dependent on the Lode angle determines the shape of the deviatoric section of the yield locus. A curve located on the octahedral plane defined by (1) for constant $\xi$ has to be periodic of period $2 \pi / 3$, and its axes of symmetry are lines $\Theta=m \pi / 3$ for $m=0,1,2$. To fulfil the mentioned properties invariant $\cos 3 \Theta$ can be used in definition of the shape function [4].

A two-parameter smooth shape function is proposed as follows:

$$
g(\Theta)=\left(1+\beta \sin ^{2} 3 \Theta\right)^{n}
$$

which is a periodic function of period $\pi / 3$ with axes of symmetry at $\Theta=m \pi / 6$ for $m=0,1,2,3,4,5 . \quad \beta$ and $n$ are free parameters which can be determined from experimental data. Definition (3) holds true for arbitrary exponent $n$ when inequality $1+\beta \sin ^{2} 3 \Theta>0$ is satisfied for every $\Theta$, what results in limitation $\beta>-1$. For $n=0$ one can get $g(\Theta)=1$, and this case is excluded from the following analysis.

Further correlation between $\beta$ and $n$ is found by imposing convexity requirement on yield function (1). Restricting attention to the following subclass of functions (1):

$$
f(\xi, r, \Theta)=\frac{r}{g(\Theta)}-h(\xi)
$$

one can specify convexity requirements on two functions $g(\Theta)$ and $h(\xi)$. Then the shape function has to fulfil the following inequality $[2,4,5]$ :

$$
g^{2}+2\left(g^{\prime}\right)^{2}-g\left(g^{\prime \prime}\right) \geq 0, \quad \text { for every } \Theta,
$$

while $-h$ has to be convex, which implies the condition [5]:

$$
h^{\prime \prime} \leq 0, \quad \text { for every } \xi \text {. }
$$

Convexity condition (5) applied to function (3) yields the following: 


$$
p(\Theta)=\left(1-36 n^{2}\right) \beta^{2} \sin ^{4} 3 \Theta+2(9 \beta n(2 n+1)+18 n+1) \beta \sin ^{2} 3 \Theta-18 \beta n+1 \geq 0 .
$$

Function (7) can attain local minima depending on the values of $\beta$ and $n$ parameters, for which the following equation is met:

$$
p^{\prime}(\Theta)=6 \beta \sin 6 \Theta\left[\left(1-36 n^{2}\right) \beta \sin ^{2} 3 \Theta+9 \beta n(2 n+1)+18 n+1\right]=0 .
$$

When $\beta=0$ function (4) defines a circle for a fixed value of $h(\xi)$, therefore convexity condition is fulfilled. If $\sin 6 \Theta=0$, then $\Theta=m \pi / 6$ for $m=0,1,2 \ldots$. Since function (3) is periodic, therefore the check of convexity can be limited to angles $\Theta=0$ and $\Theta=\pi / 6$. Moreover, the local extremum of function (7) is analysed, which is attained when:

$$
\left(1-36 n^{2}\right) \beta \sin ^{2} 3 \Theta+9 \beta n(2 n+1)+18 n+1=0 .
$$

At this level of analysis function (7) can be regarded as a quadratic function $\tilde{p}\left(\sin ^{2} 3 \Theta\right)$ being complemented by the domain conditions: $0 \leq \sin ^{2} 3 \Theta \leq 1$. Therefore, only condition for minimum of the parabola $\tilde{p}$ is considered. For $\beta \neq 0$ function $\tilde{p}$ attains its minimum if $\left(1-36 n^{2}\right) \beta^{2}>0$, i.e. for $n \in(-1 / 6,1 / 6)$, whereas for $n \in(-\infty,-1 / 6) \cup(1 / 6, \infty)$ it attains maximum. For $n= \pm 1 / 6$ function (7) has no extreme points. By combination of the formulated cases for convexity requirements, the following limits on parameters $\beta$ and $n$ are derived:

$$
\begin{gathered}
\frac{1}{18 n} \leq \beta \leq-\frac{1}{1+18 n} \quad \text { for } \quad n \in\left(-\infty,-\frac{3}{38}\right), \\
\frac{2 P-2(1+10 n)}{9 n(1+2 n)^{2}} \leq \beta \leq-\frac{2 P+2(1+10 n)}{9 n(1+2 n)^{2}} \quad \text { for } \quad n \in\left[-\frac{3}{38}, 0\right), \\
-\frac{1}{1+18 n} \leq \beta \leq \frac{1}{18 n} \quad \text { for } \quad n \in(0, \infty),
\end{gathered}
$$

with additional notation introduced:

$$
P=\sqrt{(1+n)(1+10 n)\left(1-36 n^{2}\right)}
$$

Graphical representation of the obtained limits (10) on $\beta$ and $n$ is shown in Figure 1. Indicated internal region between the presented curves defines the convexity zone for shape functions (3). 


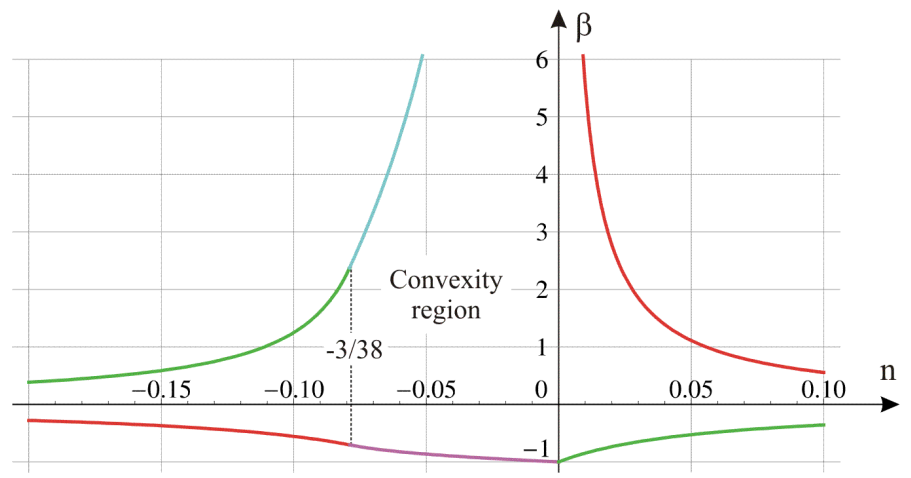

Fig. 1. Region of $n$ and $\beta$ combinations preserving convexity of deviatoric shape function (3).

\section{Application to pressure insensitive material}

As the first example of application of the introduced shape functions a pressure insensitive material is considered. Then function $h(\xi)$ is a constant function, which can be conveniently defined for tensile meridian, $\Theta=0$, or for compressive meridian, $\Theta=\pi / 3$.

Function (4) can be converted to the form:

$$
f(r, \Theta)=r-\operatorname{Rg}(\Theta)=r-R\left(1+\beta \sin ^{2} 3 \Theta\right)^{n},
$$

in which size parameter $R$ and shape parameter $\beta$ of the yield surface $f(r, \Theta)=0$ can be determined from typical experimental tests. Denoting by $r=r_{T}$ value of the second invariant for the tensile meridian, by $r=r_{C}$ for the compressive meridian, and by $r=r_{S}$ for the shear meridian, $\Theta=\pi / 6$, from (12) one can get the set of equations:

$$
r_{T}=R=r_{C}, \quad r_{S}=R(1+\beta)^{n} .
$$

Solution to (13) results in:

$$
R=r_{T}, \quad \beta=\sqrt[n]{t}-1, \quad \text { where } \quad t=r_{S} / r_{T}
$$

parameter is introduced, which describes the shape of deviatoric section of a yield surface.

Then yield function (12) can be rewritten in the form of the yield criterion:

$$
f(r, \Theta)=r-r_{T}\left[1+(\sqrt[n]{t}-1) \sin ^{2} 3 \Theta\right]^{n}=0 .
$$

Equation (15) defines a surface in the Haigh-Westergaard space which is differentiable in the domain with unique gradients. Those properties are desired for the yield or plastic potentials, and especially for numerical algorithms used in the FEM.

Parameter $t$ reflects the ratio of shear-to-axial yield stress. For the uniaxial tension test invariant $r$ is equal to $r_{T}=\sqrt{2 / 3} f_{T}$, where $f_{T}$ stands for the yield stress. In case of uniaxial compression it is $r_{C}=\sqrt{2 / 3} f_{C}$ where $f_{C}=f_{T}$. For the pure shear test the second 
invariant takes value $r_{S}=\sqrt{2} f_{S}$, with $f_{S}=k$ being the shear yield stress. Therefore for an incompressible material the ratio of shear-to-axial yield stress can be expressed as:

$$
t=\frac{\sqrt{3} k}{f_{T}}=\frac{\sqrt{3} k}{f_{C}} .
$$

When $f_{T}=\sqrt{3} k$ formula (16) gives $t=1$ and equation (15) reduces to the well-known yield criterion of Huber-Mises (HM) [2, 4]. For example, the shear-to-axial strength ratio was considered in [6] for constitutive modelling of ductile fracture of structural metals.

Using relationship (14), which relates of the shape parameter $t$ and $\beta$, namely $t=(1+\beta)^{n}$, restrictions (10) can be re-casted to $t(n)$. Hence, convexity restrictions (10) can be expressed as:

$$
\begin{gathered}
\left(1-\frac{1}{1+18 n}\right)^{n} \leq t \leq\left(1+\frac{1}{18 n}\right)^{n} \text { for } n \in\left(-\infty,-\frac{3}{38}\right) \cup(0, \infty) \\
{\left[1-\frac{2 P+2(1+10 n)}{9 n(1+2 n)^{2}}\right]^{n} \leq t \leq\left[1+\frac{2 P-2(1+10 n)}{9 n(1+2 n)^{2}}\right]^{n} \text { for } n \in\left[-\frac{3}{38}, 0\right) .}
\end{gathered}
$$

where $P(n)$ is defined by (11). Limits according to (17) and accompanying region of convexity for function (15) are shown in Figure 2. Dependence of the shape of deviatoric section on the exponent is shown in Figure 3.

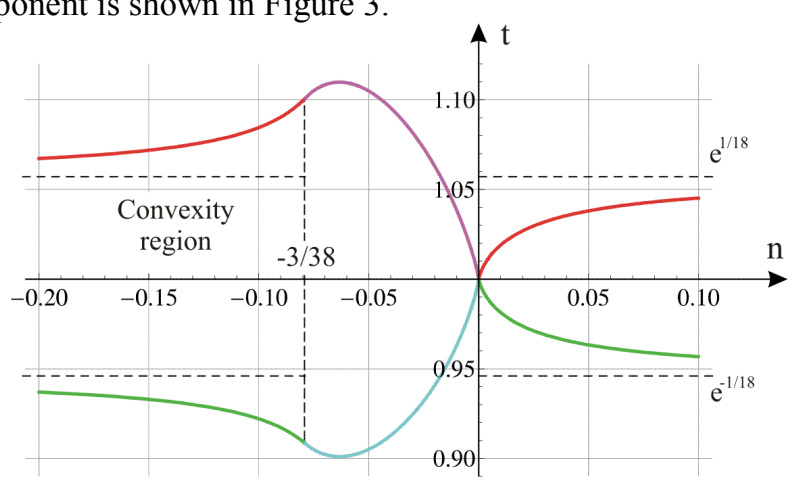

Fig. 2. Region of $n$ and $t$ combinations preserving convexity of yield criterion (15).
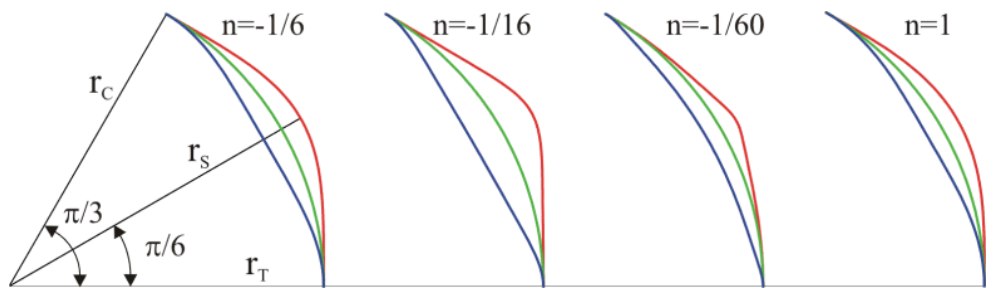

Fig. 3. Dependence of the deviatoric shape of the failure loci on the assumed values of exponent $n$ with associated limit values of $t$ and referenced to the curve for $t=1$ (HM). 
When exponent $n \rightarrow \pm \infty$, restrictions (17) imply limits $t \rightarrow \sqrt[18]{e} \cong 1.0571$ and $t \rightarrow 1 / \sqrt[18]{e} \cong 0.94596$, as shown in Fig. 2. Restrictions (17) for $t$ parameter attain local minimum or maximum, which can be found numerically from the following equation:

$$
\ln \left[1-\frac{2 P+2(1+10 n)}{9 n(1+2 n)^{2}}\right]=\frac{1+2 n+4 n(1+10 n)}{(1+2 n) P} .
$$

Extreme values of the shape parameter $t$ are attained for $n_{\mathrm{ext}}=-0.06326823$, which results in the values $t_{\min }=0.901065$ and $t_{\max }=1.109798$ (Fig. 2), or equivalently values of the associated deviatoric section shape parameter $\beta_{\min }=-0.807299$ and $\beta_{\max }=4.189380$ (Figure 1).

In case of the characteristic value of exponent $n=-3 / 38 \cong-0.0789474$ restrictions (17) and (10) yield the range $0.908436 \leq t \leq 1.100793$ with associated limits $-0.703704 \leq \beta \leq 2.375$. For close to the optimal value of exponent $n=-1 / 16$ restrictions result in the intervals: $0.901079 \leq t \leq 1.109780$ or $-0.811111 \leq \beta \leq 4.294104$. Deviatoric sections of failure criterion (15) for exponent $n=-0.0625$ are shown in Figure 4.

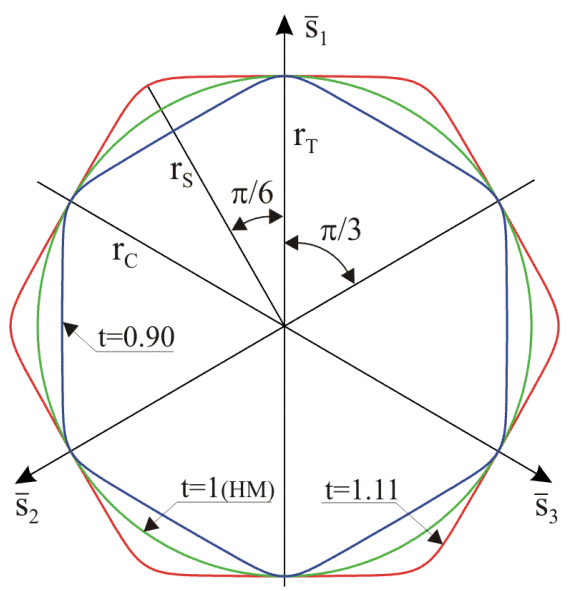

Fig. 4. Deviatoric sections of the yield criterion in the space of principal stresses for $n=-1 / 16$ and upper and lower limits for $t$ and referenced to the curve for $t=1$, where $\bar{s}_{i}=\sqrt{2 / 3} \sigma_{i}$.

Graphs of the yield criterion (15) with exponent $n=-0.0625$ and accompanying limits $t=1.109$ and $t=0.9010$ are shown in Fig. 5. When $t=0.9010$ criterion (15) can be interpreted as a smooth approximation of the well-known Tresca-Guest criterion, while for $t=1.109$ can serve as a smooth approximation of the Hill-Ivlev-Haythornthwaite surface $[3,4]$. By change of $t$ from the minimum to the maximum value a smooth transition in the shape of the yield surface can be achieved, compare Figure 6 . 
a)

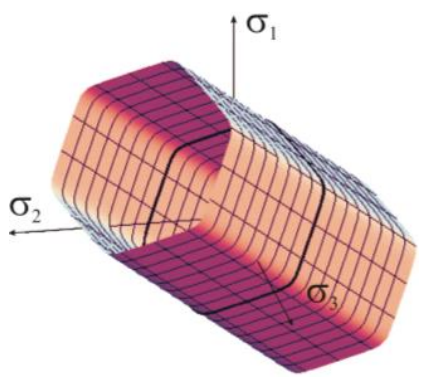

b)

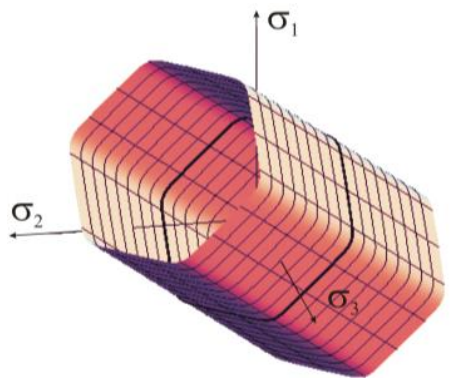

Fig. 5. Failure surfaces for $n=-1 / 16$ exponent and for the upper and lower limits of $t$ parameter.

\subsection{Plane stress}

In case of the plane stress state we use the following condition to reduce the threedimensional stress tensor $\boldsymbol{\sigma}$ to the two-dimensional counterpart $\overline{\boldsymbol{\sigma}}: \boldsymbol{\sigma}=\overline{\boldsymbol{\sigma}}+0 \mathbf{n} \otimes \mathbf{n}$ with $\overline{\boldsymbol{\sigma}}=\bar{\xi} \overline{\mathbf{k}}+\overline{\mathbf{s}}, \overline{\mathbf{k}}=\overline{\mathbf{I}} / \sqrt{2}$ [2]. Then invariants of the plane stress tensor are defined as:

$$
\bar{\xi}=\frac{1}{\sqrt{2}} \operatorname{tr} \overline{\boldsymbol{\sigma}}, \quad \bar{r}=\sqrt{\operatorname{tr} \overline{\mathbf{s}}^{2}} \quad \text { or } \quad \rho=\sqrt{\bar{\xi}^{2}+\bar{r}^{2}}, \quad \psi=\arctan \left(\frac{\bar{r}}{\bar{\xi}}\right) .
$$

Then invariants $\xi, r, \sin ^{2} 3 \Theta$ now can be reduced to the form:

$$
\xi=\sqrt{\frac{2}{3}} \rho \cos \psi, \quad r=\frac{\rho}{\sqrt{3}} \sqrt{1+2 \sin ^{2} \psi}, \quad \sin ^{2} 3 \Theta=\frac{27 \sin ^{2} \psi\left(2 \sin ^{2} \psi-1\right)^{2}}{\left(1+2 \sin ^{2} \psi\right)^{3}} .
$$

Using representation (20) yield criterion (15) can be formulated for the plane stress:

$$
\bar{f}(\rho, \psi)=\rho-\frac{r_{T} \sqrt{3}}{\sqrt{1+2 \sin ^{2} \psi}}\left[1+(\sqrt[n]{t}-1) \frac{27 \sin ^{2} \psi\left(2 \sin ^{2} \psi-1\right)^{2}}{\left(1+2 \sin ^{2} \psi\right)^{3}}\right]^{n}=0 .
$$

Graphs of criterion (21) on the plane of invariants $\bar{\xi}, \bar{r}$ or on the plane of principal stresses are shown in Figure 6. Failure surfaces in the space of components of the plane stress tensor are presented in Figure 7.
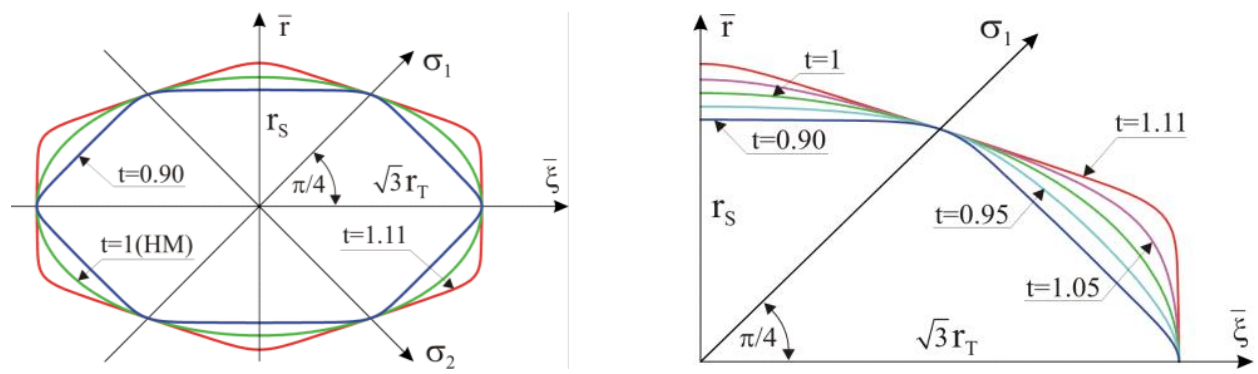

Fig. 6. Failure curves for the plane stress for $n=-1 / 16$ and several values of the shape parameter $t$. 
a)

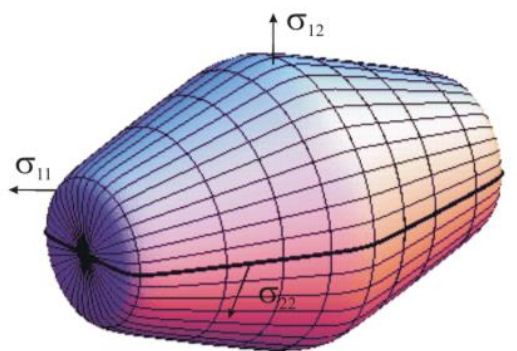

b)

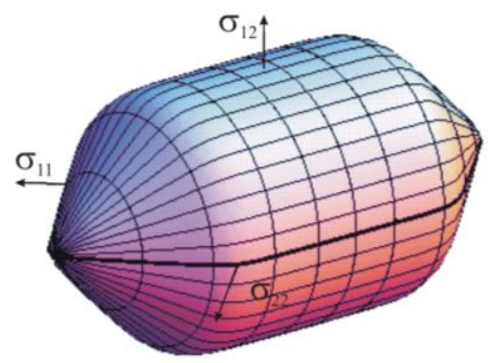

Fig. 7. Failure surfaces for the plane stress when $n=-1 / 16$ and: a) $t=1.11$, b) $\mathrm{t}=0.90$.

\section{Further applications and discussion}

Further applications of the introduced shape function for pressure sensitive materials can be done. For example, when Drucker-Prager (DP) conical surface is regarded as the case for modification in the failure function (4) function $h(\xi)$ takes the following form:

$$
h(\xi)=B-A \xi \quad \text { for } \quad \xi \leq \xi_{A},
$$

where requirement (6) is satisfied. Calibration of free parameters by usage of uniaxial tension and compression tests results in:

$$
A=\sqrt{2} \frac{\mu-1}{\mu+1}, \quad B=\sqrt{\frac{2}{3}} \frac{2 \mu}{\mu+1} f_{T}, \quad \xi_{A}=\frac{2 \mu}{\mu+1} \frac{f_{T}}{\sqrt{3}}, \quad \mu=\frac{f_{C}}{f_{T}} .
$$

Therefore, the deviatoric section shape parameter, now as well dependent on the ratio of the axial strengths $\mu$, is defined by formula:

$$
t=\frac{\mu+1}{2 \mu} \frac{\sqrt{3} k}{f_{T}} .
$$

Graphs of failure surface (4) with specification (22) is shown in Figure 8a. Failure curves for the plane stress are presented in Figure $8 \mathrm{~b}$.

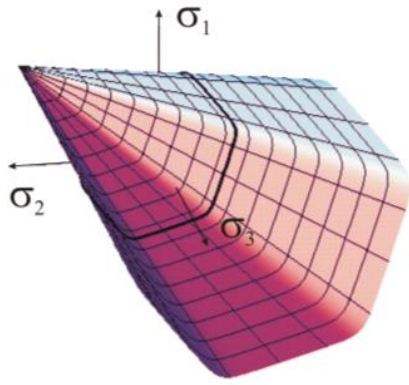

a)

Fig. 8. Modified DP criterion: a) failure surface for $t=0.9$; b) failure curves for the plane stress for $n=-1 / 16$ and $\mu=1.5$, where $\tan \omega=1 / 3$ (location of shear meridians). 
The shape functions of the same class as (3) can be defined in equivalent forms as [3]:

$$
g_{1}(\Theta)=\left(1+\alpha \cos ^{2} 3 \Theta\right)^{n} \quad \text { or } \quad g_{2}(\Theta)=(1+\gamma \cos 6 \Theta)^{n} .
$$

Function (3) is convenient in calibration when the tensile or compressive meridian in (4) is selected. Function $g_{1}$ in (25) is favourable if the shear meridian is chosen for calibration, while restrictions for $\alpha$ parameter are analogous to (10). Function $g_{2}$ has no advantages for calibration, but restrictions for $\gamma$ parameter are symmetric, compare Fig. 1 and [3, 4].

\section{References}

1. B. Raniecki, Z. Mróz, Acta Mech, 195, 81-102 (2008)

2. W.F. Chen, A.F. Saleeb, Constitutive equations for engineering materials, (Elsevier, Amsterdam, 1994)

3. A. Szwed, S. Jemioło, Theoretical Foundations in Civil Engng., V, 393-402 (1997)

4. S. Jemioło, A. Szwed, Budownictwo, 133, 5-51 (OWPW, 1999)

5. I. Kamińska, A. Szwed, Sprężystość i lepkosprężystość małych odksztatceń, 6, 79-94 (OWPW, 2017)

6. K. Nahshon, J.W. Hutchinson, Eur J Mech A/Solids, 27, 1-17 (2008) 